\title{
CONDITION MONITORING INDICATORS FOR PITTING DETECTION IN PLANETARY GEAR UNITS
}

\author{
Francesco BERLATO ${ }^{1}$, Gianluca D'ELIA ${ }^{2}$, Mattia BATTARRA ${ }^{2}$, Giorgio DALPIAZ ${ }^{2}$ \\ ${ }^{1}$ Bonfiglioli spa, Via Giovanni XXIII, 7/A - 40012 Lippo di Calderara di Reno, BO, IT \\ ${ }^{2}$ University of Ferrara, Department of Engineering, Via G. Saragat 1 - 44123 Ferrara, IT \\ giorgio.dalpiaz@unife.it
}

\begin{abstract}
In industrial field, there is an increasing demand for monitoring systems enabling predictive maintenance programs. In this context, the present work concerns the monitoring of distributed wear (pitting) in planetary gearboxes. For this purpose, some metrics of the synchronous average of the vibration signal, based on the statistical moment of the fourth order, are present in literature; in this paper, a new indicator is proposed, namely NA4mod. The effectiveness of this metric in identifying the early stage of pitting has been evaluated by conducting an accelerated life test of about 700 hours on a test bench using a back-to-back configuration. The paper introduces the proposed metric, describes the test, presents and dis-cusses the results. Metric NA4mod exhibits satisfactory capability to detect pitting with better reliability than other metrics in literature. In addition, the metric is shown to be sensitive to both early stage damage and pitting severity in the final stage. Results are verified by means of wavelet-transform analysis.
\end{abstract}

Keywords: condition monitoring, planetary gearbox, distributed wear, vibration metrics.

\section{INTRODUCTION}

Every machine with moving components gives rise to vibrations and noise; depending on its operating status, a specific "vibratory signature" characterizes each machine. It is therefore possible to detect the changes on this signature and use them as indicators of the imminent appearance of a fault. Numerous analysis procedures of the vibratory signature have been developed for condition monitoring and diagnostics, especially in the last 30 years $[1,2]$. In particular, several applications of signal processing techniques to fault detection in planetary gearboxes can be found in literature; as examples, the applications to gearboxes used in lifting systems [3], wind turbines [4] or helicopter transmissions [5] can be cited.

Signal analysis techniques can be classified according to their domain: techniques based on time, frequency or time-frequency domain. In the context of time-based techniques, an effective investigation tool for many types of defects is the Time Synchronous Average (TSA) of the signal [6, 7]. As a matter of fact, the TSA contains the signal components that are synchronous with the rotation of a reference shaft and its harmonics, while the non-synchronous and random components of the signal are heavily attenuated by the averaging operation. The TSA may be then further manipulated by applying band-pass filter that allows to isolate the mesh frequency or the rotation components. The subsequent computation of the Hilbert transform provide the chance to determine both amplitude and phase of the modulation [8-10], which typically represent an indication of the presence of faults.

Within this context, in order to obtain indexes of the presence of faults and their severity, the raw time signal or the TSA may be characterized by statistical parameters or metrics of various type [1115]. D'Elia et al. [14] have compared the performances of some condition metrics of the TSA presented in the literature [16], during an accelerated life test on planetary gearboxes, where distributed pitting developed. The obtained results show the limits of the metrics presented in the literature. In particular, metric FM4 [16] is sensitive to very heavy pitting at life end and not to the early pitting development, while metric NA4 [13, 17], seems to be sensitive to the pitting initial development and not to its growth. Thus, two new metrics of the TSA - more promising for the detection of pitting - are proposed and validated in [14], namely the Residual Variance (RV) and the Cumulative Residual Variance (CRV). Fault detection can be carried out also in the frequency domain, as described by Randall R. in ref. [18]. It is worth noting that the discrete Fourier transform can be applied to periodic stationary signals or single transients. However, localized gear defects typically introduce non-stationary components into the signal [10], which cannot be studied by conventional frequency analysis techniques. In this scenario, the adoption of advanced techniques based on the time-frequency domain such as the Continuous Wavelet Transform (CWT) $[10,19,20]$ 
and the Wigner-Ville distribution (WVD) [11, 21, 22] becomes mandatory.

The present work concerns the development of a new TSA-based metric, namely NA4mod, for pitting detection in planetary gearboxes. Such a metric is based on the fourth-order statistical moment, developed as a modification of metric NA4 introduced in Refs. [13, 17]. Both metrics are applied on two gearboxes working in back-to-back configuration with electric power recirculation, demonstrating that metric NA4mod is effectively capable to follow the evolution of the pitting severity, while the sensitivity of metric NA4 is limited to initial development of the phenomenon. The robustness of the technique is then verified by applying the Wavelet Transform on the same set of data.

The structure of the paper is as follows. In this section, the state of the art about the monitoring of distributed defects in planetary gearboxes is introduced. Section 2 details the theoretical bases of the methodology and the proposed metric. In Section 3, the experimental apparatus and the accelerated life test are described, while Section 4 reports the results about the metric effectiveness and its comparison with metric NA4. Finally, the last Section is devoted to concluding remarks.

\section{PROPOSED METRIC NA4mod AND PROCESSING METHODOLOGY}

As mentioned above, the new proposed metric is computed from the TSA of the gear of interest and, in particular, it is based both on the regular signal and the residual one [23], obtained from the TSA. Thus a short background about this processing is first given.

In the case of planetary gearboxes, the evaluation of the TSA evaluation is complicated by the movement of the planets, that causes the continuous variation of the distance between the meshing teeth and the fixed transducer mounted outside the annulus. The variation of such a relative position produces a periodic variation in the transfer function between the two components. For this reason, a specific procedure, able to eliminate the effect of the variation of the transfer functions, is needed in order to extract the TSA of each gear from the overall vibration signal. Based on this purpose, McFadden proposed a technique in the early '90s [7]. In subsequent studies, Forrester and Blunt [24] presented further modifications and improvements thanks to a specific technique that is actually covered by patents. For this reason, in the present study, devoted to industrial applications, the McFadden method has been used.

The procedure for extracting the TSA of planetary gearboxes is here shortly summarized. Firstly, by using a tachometer signal, the acceleration time signal is resampled in the angle domain according to the rotation of the carrier of the planetary stage of interest. Then, starting from the angle-based signal referred to the carrier, the signal of the tooth of the planet that meshes with the tooth of the annulus under the accelerometer is extracted one by one. In order to achieve this goal, a time window of appropriate length is used and the operation is repeated once per turn of the carrier, until the signals relating to all the teeth of the gear of interest, planet or sun, have been extracted. Finally, the operation is repeated, obtaining the signal corresponding to several turns of the gear of interest, and the signals of the different turns are averaged in a synchronous manner. A suitable number of averages should be used to obtain a high signal to noise ratio.

It is worth noting that the periodic nature of the TSA provides more possibilities to manipulation of the signal with respect to the raw vibration signal. As a matter of fact, the Fourier transform of a periodic signal, such as the TSA, consists of leakage-free discrete spectral lines suitable to be further processed by means of an ideal filter. Thus, one or more frequency lines (which here represent orders of rotation of the wheel of interest) can be completely removed from the spectrum, without causing discontinuity when the signal is one more time translated to the angle domain. This allows the application of various signal analysis techniques, specifically designed for the treatment of the TSA, and of a series of related signal metrics to be used for fault detection and diagnosis.

In particular, by filtering the TSA, the following signals can be obtained [23]: i) Regular signal: containing only the meshing frequency and its harmonics; ii) Residual signal: obtained from the TSA, eliminating the meshing frequency and its harmonics, as well as the rotation frequency and its second harmonic in the low frequency range.

One of the metrics based on the fourth-order statistical moment of the TSA is the NA4 metric, already introduced in literature [13, 17], which is defined as the ratio between the fourth order statistical moment of the residual signal and the current average of the square of the variance of the residual signal itself (the averaging process is performed on all the records prior to and including the current measurement):

$$
N A 4=\frac{\frac{1}{N} \sum_{i=1}^{N}\left(r_{i M}-\bar{r}_{M}\right)^{4}}{\frac{1}{M} \sum_{j=1}^{M}\left[\frac{1}{N} \sum_{i=1}^{N}\left(r_{i j}-\bar{r}_{j}\right)^{2}\right]^{2}}
$$

where $r$ is the residual signal, $\bar{r}$ is the mean value of the residual signal, $j$ is the record index, $i$ is the sample index, $M$ is the number of current records and $N$ is the number of sample in the time records.

The new metric proposed in this work, indicated as NA4mod, has been developed as a modification of NA4, with the purpose to obtain an indication related to the severity of the fault, even when such a fault passes from localized to distributed. Thus, the new metric is characterized by the normalization of the fourth statistical moment of the residual signal 
with respect to the current average of the square of the variance of the regular signal (this average is calculated on all the records prior to and including the current measurement):

$$
N A 4 \bmod =\frac{\frac{1}{N} \sum_{i=1}^{N}\left(r_{i M}-\bar{r}_{M}\right)^{4}}{\frac{1}{M} \sum_{j=1}^{M}\left[\frac{1}{N} \sum_{i=1}^{N}\left(s r_{i j}-\overline{s r}\right)^{2}\right]^{2}}
$$

where $s r$ is the regular signal and $\overline{s r}$ is the mean value of the regular signal. Such a normalization to the regular signal is finalized to account for the specific meshing condition.

\section{EXPERIMENTAL APPARATUS AND ACCELERATED LIFE TEST}

In order to assess the capabilities of metric NA4mod, a dedicated set of data is firstly achieved. The experiment has been designed to produce a real case of damage, including the complete development of pitting, without compromising the functionality of the gearbox and the test bench.

This end-of-life test has been performed by using two Bonfiglioli gearboxes model 304 L3 220 P CP100, called respectively 'Gearbox A' (GB A) and 'Gearbox B' (GB B), in back-to-back configuration with electric power recirculation (Fig. 1.a). Based on this layout, one of the two gearboxes, GB B, works as a speed multiplier, while the other, GB A, works as a speed reducer. The two identical gear units are connected through the output shaft by means of a double universal joint. The main gearbox data are shown in Tab. 1, while the gearbox scheme is given in Fig. 1.b.

Table 1. Gearbox data

\begin{tabular}{|l|c|c|c|}
\hline Data & Stage 1 & Stage 2 & Stage 3 \\
\hline Sun tooth number & 10 & 10 & 24 \\
\hline Planet tooth number & 25 & 25 & 26 \\
\hline $\begin{array}{l}\text { Annulus tooth } \\
\text { number }\end{array}$ & 62 & 62 & 78 \\
\hline Number of planets & 3 & 3 & 4 \\
\hline Stage ratio & 7.2 & 7.2 & 4.25 \\
\hline Total ratio & \multicolumn{3}{|c|}{220.32} \\
\hline
\end{tabular}

Each gearbox is driven by a $4 \mathrm{~kW}$ asynchronous electric motor (Bonfiglioli BN 112 L4) equipped with a 1024-line Sick incremental encoder, used for feedback control. In order to effectively manage the power, two Bonfiglioli Active-CUBE inverters are used. The first one drives the electric motor related to gearbox GB A, controlling the speed of the test bench through its encoder. The second inverter, on the contrary, controls the motor connected to GB B, which actually works as a current generator. The inverter allows to regulate the current absorption.

Two B\&K 4507 piezoelectric accelerometers are positioned onto the casings of the two gearboxes, close the second stage annulus gear. The vibration signals are continuously acquired at a sampling rate of $25600 \mathrm{~Hz}$ by using National Instruments NI9234 card. The tachometer signal is obtained through an inverter function, which duplicates the encoder signal by reducing the number of lines by $1 / 30$, according to the capacity of acquisition card. In order to speed up the end-oflife test, the speed multiplier GB B was filled with the standard lubricant indicated in the data sheet, while a lower viscosity lubricant was used in GB A, object of the damage test. In addition, the load was increased beyond the nominal one, in order to promote wear development. The end-of-life test has been carried out for about 700 hours at the constant speed of $1500 \mathrm{rpm}$, with a torque equal to 4400 $\mathrm{Nm}$, i.e. $30 \%$ higher than the nominal torque reported in the data sheet for this speed.

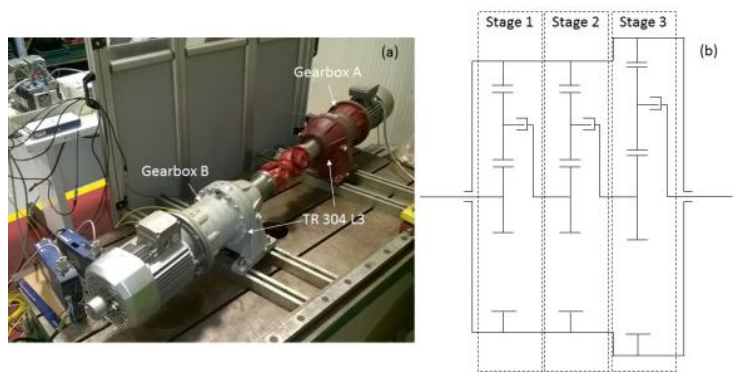

Fig. 1. Test bench setup (a) and internal gearbox scheme (b)

At the end of the test, the gearboxes were inspected. The effects of the accelerated fatigue test may be easily recognized on the sun gear of the second stage of GB A (see Fig. 2.a), that presents distributed pitting and strong upsetting in the contact area, due to the meshing with the planet gears. Damages affecting the head profile and the non-meshing side (crushing) have been also detected. Analysing GB B, only slight pitting was found at the base of the teeth on the sun gear of the second stage (see Fig. 2.b).
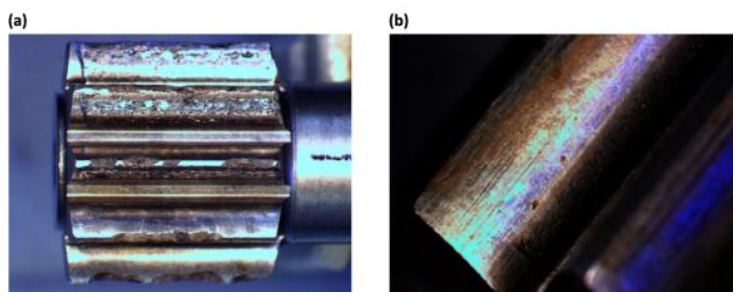

Fig. 2. Detailed view of the wear on the teeth of the sun gear of the second stage of (a) GB A and (b) GB B

\section{RESULTS AND DISCUSSION}

The present Section details results concerning the proposed metric NA4mod in comparison with metric NA4. The analysis is hereinafter performed on the sun gear of the second stage for both gearboxes, although the metrics have been also computed for the TSA of the planets and the sun of all the stages.

As a first step, the trends of the most common statistical parameters, i.e. RMS values and kurtosis 

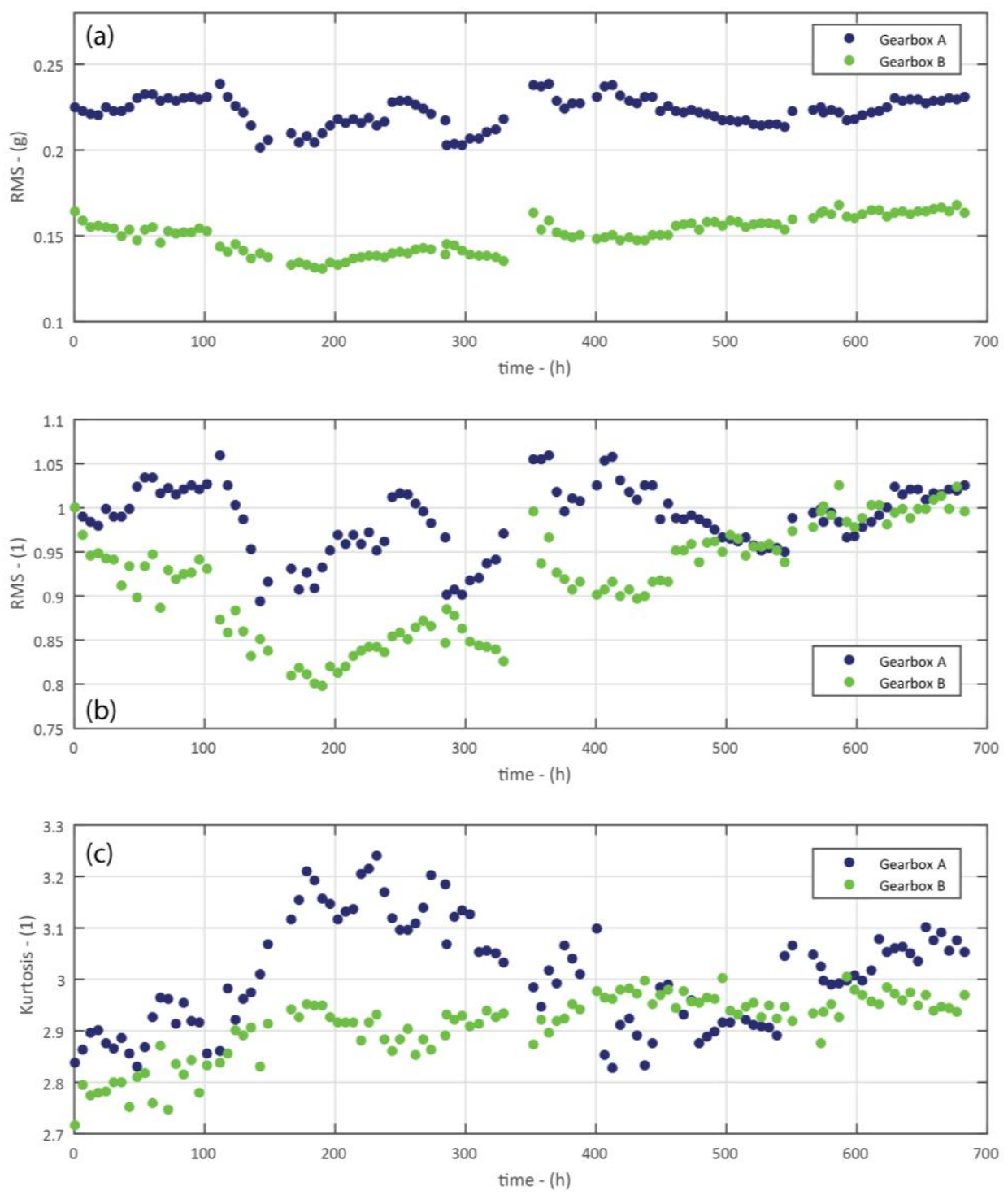

Fig. 3. RMS (a), RMS normalized values with respect to the first record (b) and kurtosis of the raw vibration signal (c) for GB A (blue) and GB B (green)

values, have been considered. They have been evaluated from the raw signals in time domain measured by the accelerometers mounted on the casings, close to the second stage annulus gears. As it is well known, RMS is related to the energetic content of the signal, while kurtosis is sensitive to impulsive contents. Figure 3 shows the result of this analysis. The RMS values concerning GB A are greater than those of GB B (Fig. 3.a) for the whole test; this effect could be related to the different viscosity of the lubricant in the two gearboxes. In order to better compare the RMS trends with reference to variations due to faults, Fig. 3.b depicts RMS values after normalization with the value of the first record of the test: the trends are oscillating until 500 hours, while they are comparable at the end of the test. Thus, from this result, no evidence of faults can be detected.

The results about kurtosis, reported in Fig. 3.c, gradually increases during the test from about 2.7 to
3.0 for gearbox GB B, while kurtosis values referred to GB A slightly increase until 3.25 in the range of 150-300 hours and in the following period they exhibit lower values, between 2.8 and 3.1, which are comparable to those of GB B. This increase in the 150-300 hours range could be related to the initial development of a localized fault, producing impulsive contents in the time signal that are detected by the kurtosis. However, as the fault grows and becomes more distributed, the impulsive content decreases and the kurtosis values fall down. Therefore, this statistic may be able to detect the initial development of localized faults, but it cannot follow the subsequent propagation and the actual severity.

Figure 4 reports the trends of metric NA4mod computed for the two gearboxes. As it may be observed, NA4mod metric related to gearbox GB A exhibits a sudden increment within the 150-200 hours interval, which is not recognized in the 
results related to GB $\mathrm{B}$. This increment is an index of the possible triggering of the pitting in GB A. hours (index of the possible triggering of the pitting), but the difference between GB A and GB

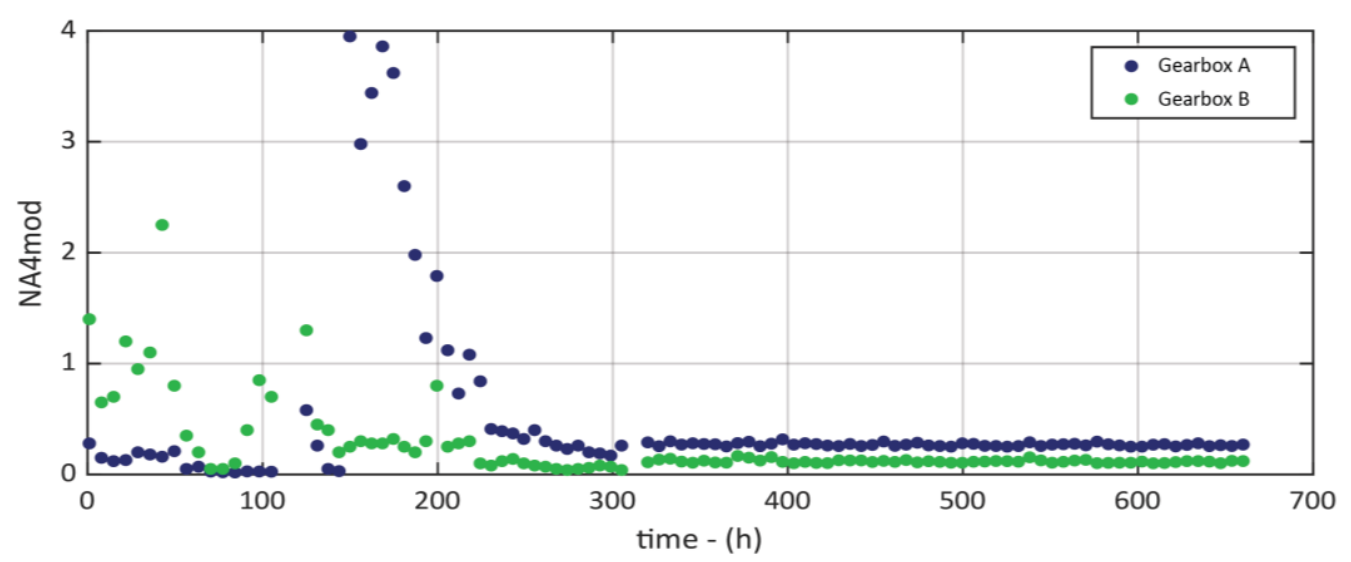

Fig. 4. NA4mod metric calculated on the TSA of the sun gear of the second stage for GB A (blue) and GB B (green)

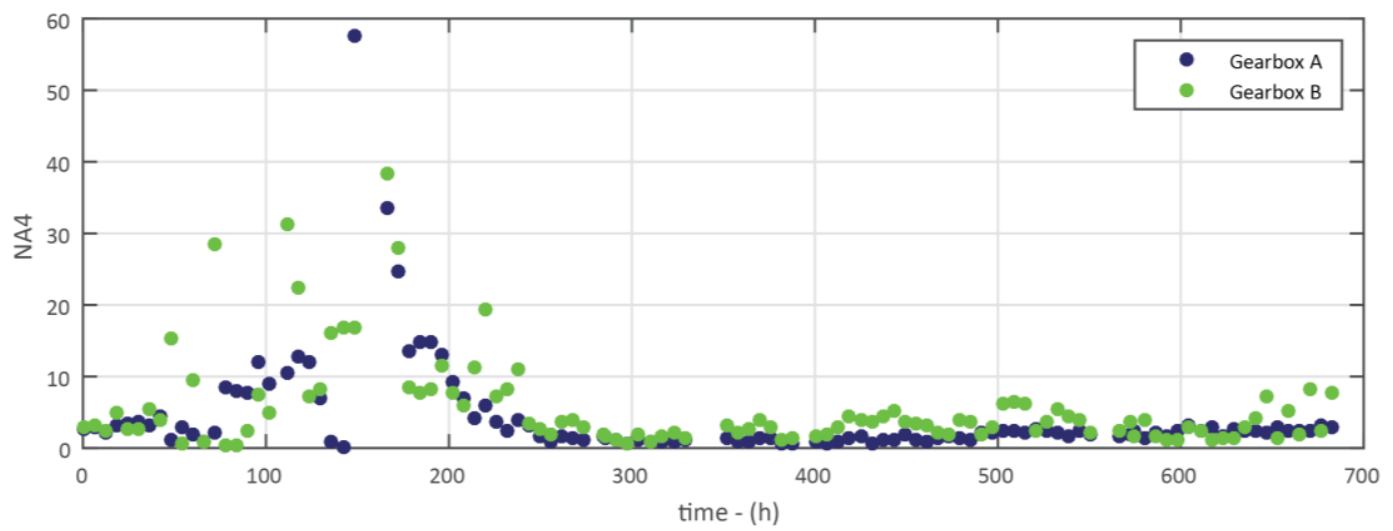

Fig. 5. NA4 metric calculated on the TSA of the sun gear of the second stage for GB A (blue) and GB B (green)

Furthermore, this parameter proves to be sufficiently robust to monitor the progress of the defect, since its value related to GB A remains always higher than that related to GB B after the defect appearance. This aspect is particularly effective in the last part of the test, which is characterized by heavy, distributed pitting. The slight difference detected between the values of NA4mod for the two gearboxes at the end of the test is the result of the condition of wear of the two sun gears, which were very different. For the sake of completeness, it has to be observed that the described trend is reversed at the beginning of the test, throughout the first 80 hours, despite the absence of damages on both gearboxes. This aspect, which represents limitation of the proposed metric, is actually smoothed by the fact that the computed values are still below the increment observed in the 150-200 hours range.

In order to assess the capabilities of metric NA4mod, Fig. 5 reports the trends of metric NA4 for the two gearboxes, concerning the same sun gears of the second stage. As it may be observed, such a metric exhibits an increment between 150 and 200
$\mathrm{B}$ is not easily recognizable as for metric NA4mod. Moreover, in contrast with respect to NA4mod, metric NA4 cannot distinguish the conditions of GB $\mathrm{A}$ and GB $\mathrm{B}$ at the end of the test. As a matter of fact, metric NA4 is not sensitive to the pitting severity in the last part of the test and therefore it is not capable to monitor the progress of the defect.

The described behavior is confirmed by the Wavelet Transform analysis of the TSA signal of the sun gear of the second stage for GB A and GB B reported in Fig. 6 and 7, respectively. Each figure is computed by considering the range between the $40^{\text {th }}$ and $50^{\text {th }}$ order and it depicts three conditions during the test: start of the test, at 150 hours, end of the test. As it may be appreciated, at the test inception (Fig. 6.a) gearbox GB A exhibits higher signal amplitude then GB B even though Fig. 6.b demonstrates that the trigger of localized pitting occurs at around 150 hours in GB A. At the end of the test (Fig. 6.c), GB A signal presents higher distributed amplitude in range 40-50 orders, in agreement with the results achieved with metric NA4mod. 

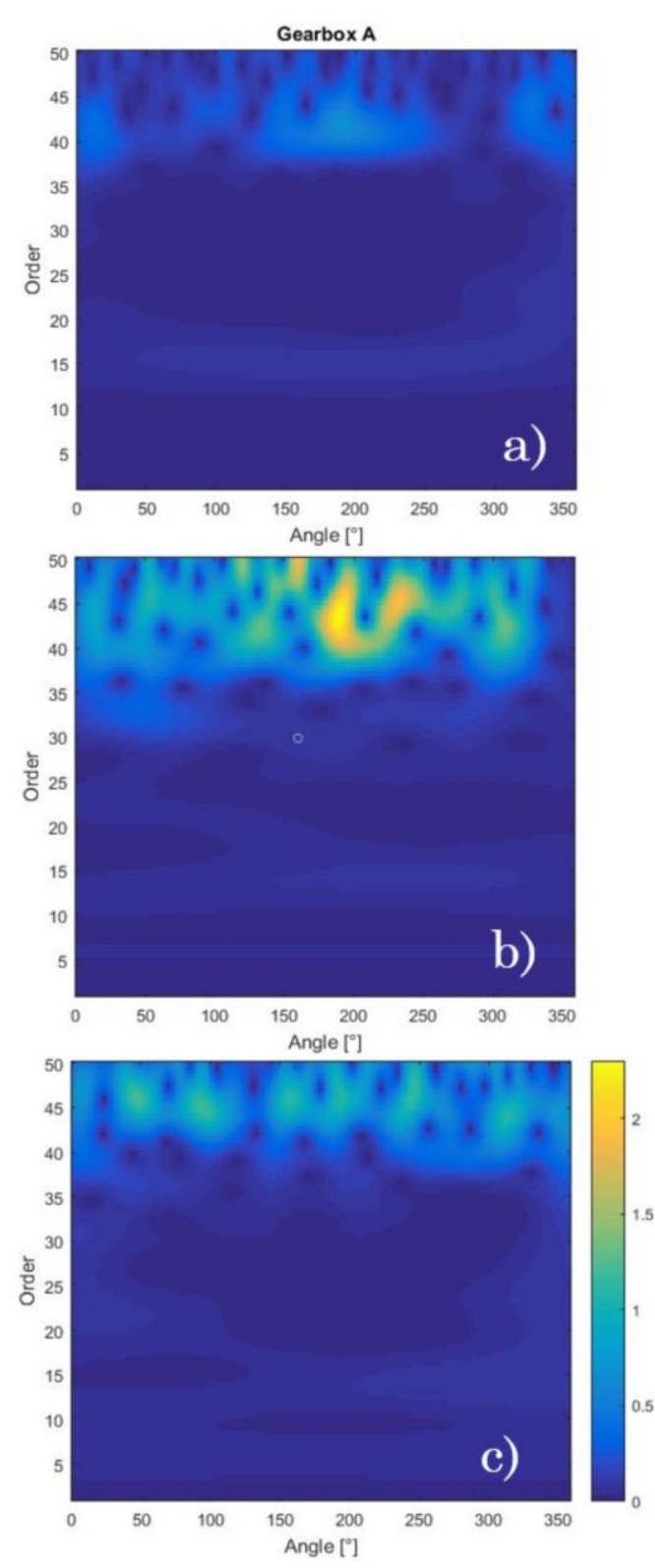

Fig. 6. Wavelet Transform calculated on the TSA of the sun gear of the second stage for GB A in three different conditions: (a) start of the test, (b) at 150 hours, (c) end of the test

\section{CONCLUDING REMARKS}

The present study has described a new metric of the TSA for pitting detection in planetary gearboxes and its effectiveness is evaluated through an accelerated fatigue test, specifically designed to promote the pitting phenomenon on the sun gear. The test follows the entire pitting evolution until the gearbox functionality is effectively compromised. The proposed metric, NA4mod - defined as the ratio between the statistical moment of the fourth order of the residual signal and the current mean of the square of the variance of the regular signal - has been developed as a modification of NA4, with the aim to obtain an indication related to the severity of
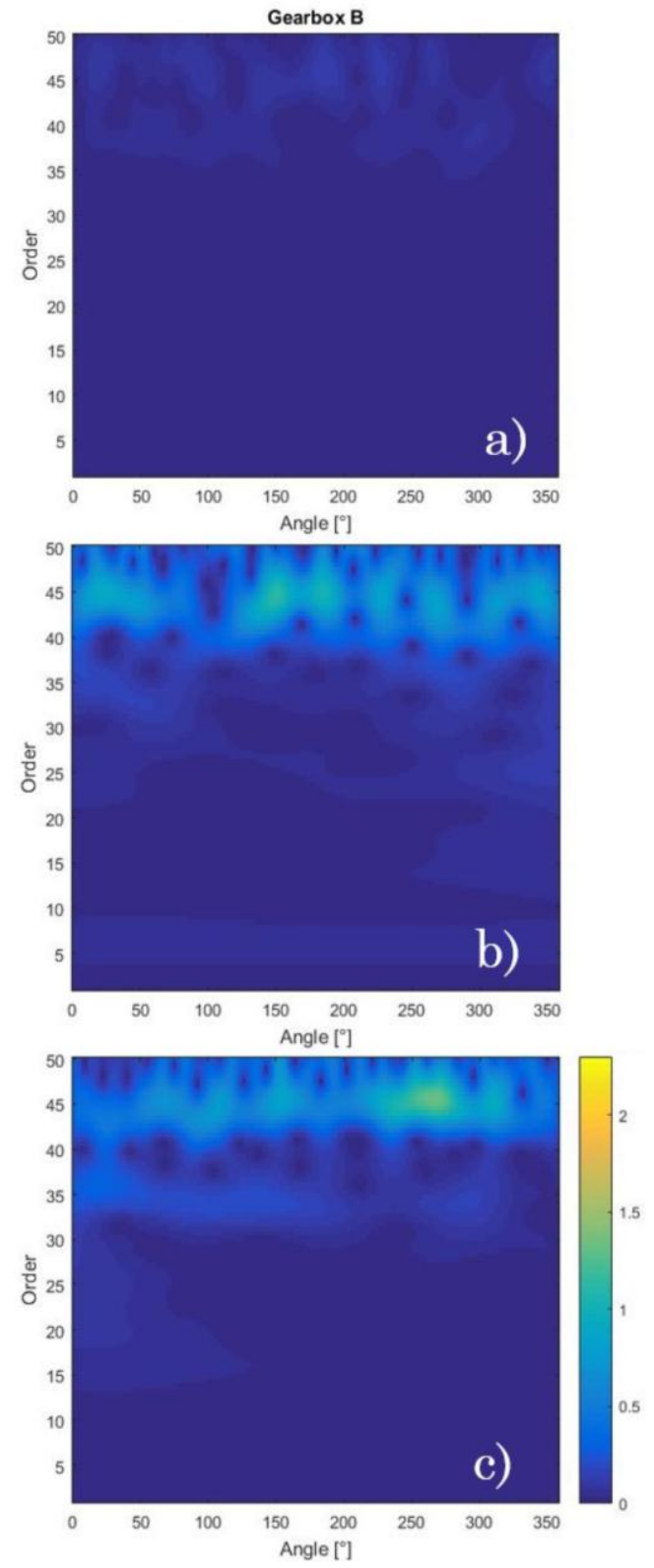

Fig. 7. Wavelet Transform calculated on the TSA of the sun gear of the second stage for GB B in three different conditions: (a) start of the test, (b) at 150 hours, (c) end of the test

the fault, even when it progressively switches from localized to distributed.

Metric NA4mod is shown to provide satisfactory detection capabilities, with greater reliability than other metrics proposed in the literature. As shown by the computed trends, the metric exhibits a sudden increment at the triggering of the pitting and it later follow the pitting severity, even though its sensitivity is slightly downgraded. In order to obtain a comprehensive evaluation of the metric sensitiveness, the results are compared with the RMS and the kurtosis values of the raw signal in time domain and with metric NA4 of the TSA. In this context, the analysis demonstrates that the RMS does not exhibit fault detection capability in 
this application, while Kurtosis detects the initial development of a localized fault but not its propagation and severity. Metric NA4 is sensitive to the pitting initial development, but, in contrast with respect to NA4mod, it is not sensitive to its severity in the final stage. The correctness of the analysis is finally verified by calculating the Wavelet Transform of TSA signals.

Based on this analysis, the proposed metric may represent a useful indicator for pitting monitoring in industrial environment, in which advanced techniques, such as analyses in the Time-Frequency domain and EMD-based algorithms, may not be suitable due to the high computational costs related.

\section{REFERENCES}

1. Smith JD. Gear Noise and Vibration. CRC Press, 1999.

2. Jardine AK, Lin D, Banjevic D. A review on machinery diagnostics and prognostics implementing condition-based maintenance, Mechanical Systems and Signal Processing. 2006; 20(7):1483-1510.

https://doi.org/10.1016/j.ymssp.2005.09.012.

3. Assaad B, Eltabach M, Antoni J. Vibration based condition monitoring of a multistage epicyclic gearbox in lifting cranes, Mechanical Systems and Signal Processing. 2014;42(1):351-367. https://doi.org/10.1016/j.ymssp.2013.06.032

4. Sawalhi N, Randall RB. Gear parameter identification in wind turbines using diagnostic analysis of gearbox vibration signals, in: Advances in Condition Monitoring of Machinery in NonStationary Operations. 2014:273-285.

5. Decker HJ, Lewicki DG. Spiral bevel pinion crack detection in a helicopter gearbox. Tech. report, NASA. 2003.

6. McFadden P. Examination of a technique for the early detection of failure in gears by signal processing of the time domain average of the meshing vibration. Mechanical Systems and Signal Processing. 1987;1(2):173-183.

https://doi.org/10.1016/0888-3270(87)90069-0.

7. McFadden P. A technique for calculating the time domain averages of the vibration of the individual planet gears and the sun gear in an epicyclic gearbox. Journal of Sound and Vibration. 1991; 144(1):163172. https://doi.org/10.1016/0022-460X(91)90739-7.

8. McFadden PD. Detecting fatigue cracks in gears by amplitude and phase demodulation of the meshing vibration. Journal of Vibration and Acoustics. 1986; 108(2):165-170. https://doi.org/10.1115/1.3269317.

9. Diez-Ibarbia A, Battarra M, Palenzuela J, et al. Comparison between transfer path analysis methods on an electric vehicle. Applied Acoustics. 2017;118 (Suppl. C):83-101.

10. Dalpiaz G, Rivola A, Rubini F. Effectiveness and sensitivity of vibration processing techniques for local fault detection in gears. Mechanical Systems and Signal Processing. 2000;14(3):387-412. https://doi.org/10.1006/mssp.1999.1294.

11. Polyshchuk V, Choy F, Braun M. Gear fault detection with time-frequency based parameter np4. International Journal of Rotating Machinery, 2002;8 (1): $57-70$.

12. Battarra M, Mucchi E. Incipient cavitation detection in external gear pumps by means of vibro-acoustic measurements. Measurement, 2018;129: 51-61. https://doi.org/10.1016/j.measurement.2018.07.013.

13. Decker HJ, Handshuh R, Zakrajsek J. An Enchancement to the NA4 Gear Vibration Diagnostic Parameter, Tech. report, NASA, 1994.

14. ReG. D'Elia, M. Cocconcelli, R. Rubini, G. Dalpiaz, Evolution of gear condition indicators for diagnostics of planetary gearboxes, in: Proceeding of International Conference Surveillance 8, 2015.

15. Samuel PD, Pines DJ. A review of vibration-based techniques for helicopter transmission diagnostics. Journal of Sound and Vibration. 2005;282(1):475508. https://doi.org/10.1016/j.jsv.2004.02.058.

16. Zakrajsek J. Some useful data analysis techniques for gearbox diagnostics. Tech. report, Institute of Sound and Vibration Research. University of Southampton, 1977.

17. Zakrajsek J. An Investigation of Gear Mesh Failure Prediction Techniques, Tech. report, NASA. 1989).

18. Randall RB. Vibration based condition monitoring, John Wiley \& Sons Inc, 2011.

19. Wang W, McFadden P. Application of wavelets to gearbox vibration signals for fault detection, Journal of Sound and Vibration. 1996; 192(5):927-939. https://doi.org/10.1006/jsvi.1996.0226.

20. Bendjama H, Bouhouche S, Boucherit $M$. Application of wavelet transform for fault diagnosis in rotating machinery, International Journal of Machine Learning and Computing. 2012; 2(1):82-87.

21. Martin W, Flandrin P. Wigner-Ville spectral analysis of nonstationary processes. IEEE Transactions on Acoustics, Speech, and Signal Processing. 1985;33(6):1461-1470. https://doi.org/10.1109/TASSP.1985.1164760.

22. Staszewski W, Worden K, Tomlinson G. Timefrequency analysis in gearbox fault detection using the Wigner-Ville distribution and pattern recognition, Mechanical Systems and Signal Processing. 1997; 11(5):673-692. https://doi.org/10.1006/mssp.1997.0102.

23. McClintic K, Lebold M, Maynard K, Byington C, Campbell R. Residual and difference feature analysis with transitional gearbox data, in: 54th Meeting of the Society for Machinery Failure Prevention Technology. 2000.

24. Forrester D, Blunt D. Analysis of epicyclic gearbox vibration, in: Proceedings of the DSTO Third International Conference on Health and Usage Monitoring - HUMS2003. 2003;5(10).

\section{Received 2019-10-14}

Accepted 2020-01-03

Available online 2020-01-07

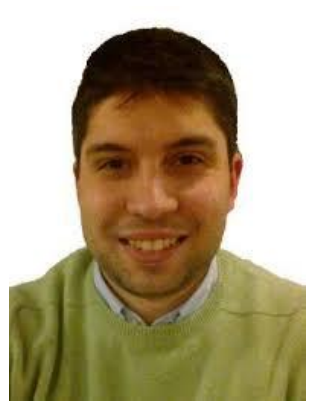

Francesco BERLATO has got his Ph.D. in Signal Processing and Machine Diagnostics in April 2017 and he is currently employed as Tools \& Models Developer for Mechatronics \& IoT Area at the Bonfiglioli Riduttori Product and Research Center after three years working as Condition Monitoring and Testing Specialist for the same company. 


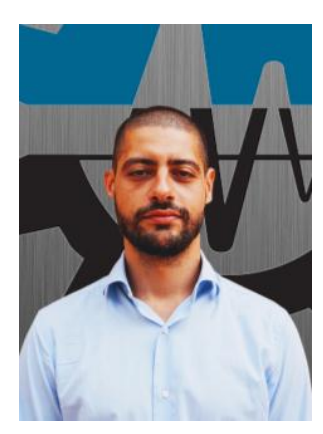

Gianluca D'ELIA is Assistant Professor at the Engineering Department of the University of Ferrara and lecturer of Diagnostics of rotating machinery. His research activity is mainly in the field of signal processing for the diagnostics of rotating machines and experimental vibration measurements. In particular, his work is focused on time-frequency analysis and Cyclostationary analysis.

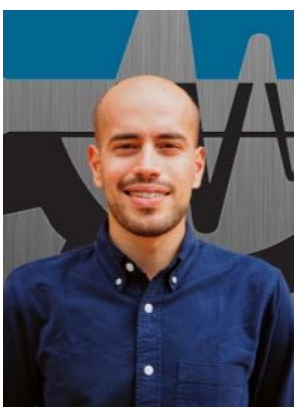

Mattia BATTARRA is PostDoc Research Assistant at the Engineering Department of the University of Ferrara since February 2018, when he received the $\mathrm{PhD}$ in Mechanical Engineering from the University of Ferrara, obtaining the "Department of Engineering UNIFE Best PhD Thesis Award 2018". After receiving the BSc and MSc from the same University, he took part to the European project COST Action TU1105 as a visiting research student at the Loughborough University, UK, while in 2016, he has been a Visiting Scholar at The Ohio State University, $\mathrm{OH}$ - US.

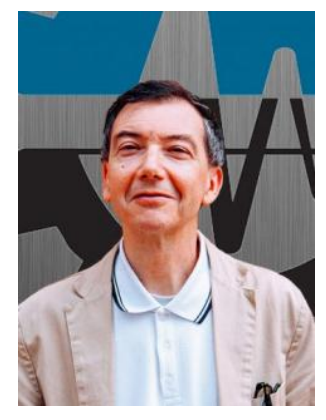

Giorgio DALPIAZ is full professor of Mechanics of Machines and Vibrations at the University of Ferrara, Department of Engineering, since September 2000. He is currently lecturer of the course of Mechanics of Vibrations in the Master Degree of Mechanical Engineering and the courses of Mechanics of Machines and Drive Mechanics of the Bachelor Degree of Mechanical Engineering. He has authored and co-authored more than 140 papers, mostly published in international journals or international conference proceedings and he is the coordinator of numerous projects funded by regional, national and EU grants. 